\title{
Effects of Blade Parameters on the Flow Field and Classification Performance of the Vertical Roller Mill via Numerical Investigations
}

\author{
Chang Liu, ${ }^{1}$ Zuobing Chen, ${ }^{1}$ Weili Zhang, ${ }^{2}$ Chenggang Yang, $^{2}$ Ya Mao, ${ }^{1}$ Yongjie Yu, \\ and Qiang Xie $\mathbb{D}^{1}$ \\ ${ }^{1}$ School of Mechanical and Electronic Engineering, Wuhan University of Technology, Wuhan 430070, China \\ ${ }^{2}$ Hefei Zhongya Building Material Equipment Co., Ltd, Hefei 230601, China \\ Correspondence should be addressed to Qiang Xie; q.xie@whut.edu.cn
}

Received 30 July 2020; Revised 14 October 2020; Accepted 19 October 2020; Published 4 November 2020

Academic Editor: Babak Shotorban

Copyright (c) 2020 Chang Liu et al. This is an open access article distributed under the Creative Commons Attribution License, which permits unrestricted use, distribution, and reproduction in any medium, provided the original work is properly cited.

\begin{abstract}
The vertical roller mill is an important crushing and grading screening device widely used in many industries. Its classification efficiency and the pressure difference determine the entire producing capacity and power consumption, respectively, which makes them the two key indicators describing the mill performance. Based on the DPM (Discrete Phase Model) and continuous phase coupling model, the flow field characteristics in the vertical roller mill including the velocity and pressure fields and the discrete phase distributions had been analyzed. The influence of blade parameters like the shape, number, and rotating speed on the flow field and classification performance had also been comprehensively explored. The numerical simulations showed that there are vortices in many zones in the mill and the blades are of great significance to the mill performance. The blade IV not only results in high classification efficiency but also reduces effectively the pressure difference in the separator and also the whole machine. The conclusions of the flow field analysis and the blade effects on the classification efficiency and the pressure difference could guide designing and optimizing the equipment structure and the milling process, which is of great importance to obtain better overall performance of the vertical roller mill.
\end{abstract}

\section{Introduction}

The vertical roller mill, integrating various processes including crushing, grinding, drying, conveying, and powder selection $[1,2]$, is broadly employed in many fields like cement, electric power, metallurgy, chemical, and nonmetallic ore industries. However the vertical roller mill, as a common choice due to its multiple functions, always consumes much energy in the whole production line. For example, according to statistics, the energy consumption of ore crushing accounts for $30 \%-50 \%$ of the total production energy consumption [3-6]. In the vertical roller mill, the fan provides a negative pressure environment and powder conveying gas. Improving classification efficiency and reducing pressure difference can effectively reduce the energy consumption of the fan. Therefore, some related studies have been conducted focusing on the variables and mechanisms affecting energy consumption.

Many scholars have studied the flow field characteristics of vertical roller mill. Sun et al. [7] analyzed the three-dimensional flow of the chamber using the standard k-epsilon turbulent model, RNG k-epsilon turbulent model, and Reynolds stress equation model (RSM). The results show that the high-pressure region improves the transportation efficiency of powder. Bhasker et al. [8] explained the meshing method and analyzed the gas-solid two-phase flow field of the vertical roller mill and finally obtained the detailed movement track of the gas-solid two-phase flow from the inlet to the outlet. Vuthaluru et al. [9] used a granular Eulerian-Eulerian approach to carry out a multiphase flow simulation of a simplified model. Dou et al. [10] adopted the CFD (Computational Fluid Dynamics) technology to 
simulate the flow field of the vertical roller mill and studied the reasons for the large pressure difference in the mill.

To explore the influence of structural and operational parameters on the flow field, Ze et al. [11] considered the effect of different gas fluxes on the gas-solid coupling flow field. The results showed that the gas flux is directly proportional to the pressure difference, and it is also pointed out that the structure has a greater influence on the flow field than the gas flux. Huang et al. [12] explored the influence of several guide blade structures on the flow field of the turbo air classifier based on fluent. The results indicated that the guide blade structure has a significant impact on the flow. Ren et al. [13] analyzed the influence of the rotor blade profile and the field in a turbo air classifier. Simulation results indicate the significant improvement of flow field distribution in the rotor cage with nonradial arc blades. Liu et al. [14] simulated the inner flow field of different structures. The simulation results show that axial inclined guide vanes can decrease the upward axial velocity in the annular region. It is found that the blade parameters have a great influence on the flow field.

To investigate the influence of operation and structure parameters on classification performance, Shah et al. [15] studied the effect of different blade angles on the classification efficiency and obtained that the optimal blade angle was $65^{\circ}$. Eswaraiah et al. [16] displayed the effects of air volume, feed rate, and blade angle on the classification performance of air classifiers and obtained the optimal value. Li et al. [17] explored the effect of air volume on the classification performance of the vertical roller mill through experimental methods. The results showed that the classification efficiency dropped significantly when the wind speed was small. Zeng et al. [18] studied the influence of the rotor cage speed and air velocity on the classification performance of turbo air classifier via numerical simulation, and two process parameters were optimized. Huang et al. [12] investigated the effects of several guide vanes on the classification performance of the turbo air classifier based on CFD technology and found that the positively bowed guide blade could improve classification precision. Caliskan et al. [19] investigated efficiency, pressure losses, and triple classification potential of the cyclone classifier at different flow rates and configurations. The results show that efficiency and pressure loss increase as the velocity increases. It can be indicated that the blade parameters have an important influence on the classification performance of the classifier. It should be noted that although the structure of these classifiers is different, their classification principles are similar. Therefore, it can be inferred that the blade parameters are of great significance to the performance of a vertical roller mill.

Generally speaking, the literation study shows the research on the vertical roller mill like the powder separation mechanisms, and the flow characteristics are still incomplete and not comprehensive. The blades especially, as key parts in the roller mill, have not been comprehensively investigated. Deep understandings of the relationships and mechanisms between the blades and the mill performance could provide a theoretical basis for designing, running, controlling, and optimizing a vertical roller mill, reducing eddy current and energy consumption, and obtaining better equipment performance with high efficiency and low resistance.

As a consequence, this work focuses on the blades in an industry-used vertical roller mill. Benefiting from the development of computer technology and numerical modeling techniques especially the CFD simulations, the numerical simulation method is applied as many other works did. A corresponding numerical model has been established by coupling the continuous phase and the DPM model. The flow characteristics in the mill were comprehensively explored via ANSYS FLUENT Release 17.0. The influences of the blade variables like shape, number, and rotating speed on the flow field and the classification performance were analyzed and discussed. The organization of this paper is as follows. The numerical model and geometry model are described in Section 2. The simulation results of flow field characteristics and classification performance are shown and discussed in Section 3. And Section 4 gives the conclusions.

\section{Model Description}

2.1. Governing Equation. The fluid flow in a vertical roller mill must follow the law of physical conservation. The energy conservation equation is ignored due to the no exchange issue involved. The three-dimensional steady simulation was performed using ANSYS FLUENT commercial software package. The flow of gas and particles obeys the conservation of mass, and the conservation equation is as follows [20]:

$$
\frac{\partial \rho}{\partial t}+\frac{\partial(\rho u)}{\partial x}+\frac{\partial(\rho v)}{\partial y}+\frac{\partial(\rho w)}{\partial z}=0
$$

The divergence is introduced in

$$
\begin{array}{r}
\nabla(\rho U)=\frac{\partial(\rho u)}{\partial x}+\frac{\partial(\rho v)}{\partial y}+\frac{\partial(\rho w)}{\partial z}, \\
\frac{\partial \rho}{\partial t}+\nabla(\rho U)=0,
\end{array}
$$

where $\rho$ is the fluid density; the fluid density is constant when fluid is incompressible. $U$ is the speed vector. $u, v$, and $w$ are the components of $U$ along $x, y$, and $z$ directions, respectively.

The movements of air and particles obey the law of conservation of momentum [20]. The equation of conservation of momentum in each direction is established as

$$
\begin{gathered}
\frac{\partial(\rho u)}{\partial t}+\nabla(\rho u U)=\nabla(\rho \nabla u)-\frac{\partial P}{\partial x}+F_{x}, \\
\frac{\partial(\rho v)}{\partial t}+\nabla(\rho v U)=\nabla(\rho \nabla v)-\frac{\partial P}{\partial y}+F_{y}, \\
\frac{\partial(\rho w)}{\partial t}+\nabla(\rho w U)=\nabla(\rho \nabla w)-\frac{\partial P}{\partial z}+F_{z},
\end{gathered}
$$

where $U$ presents fluid velocity, and $U=u \vec{i}+v \vec{j}+w \vec{k} \cdot P$ is the pressure on the fluid element. $F_{x}, F_{y}$, and $F_{z}$ are the 
force component acting on the $x, y$, and $z$ directions of the infinitesimal body.

At present, the most commonly used discrete schemes are first-order upwind scheme, second-order scheme, and QUICK scheme. The first-order upwind scheme has a lower truncation level and accuracy. The second-order upwind scheme is based on the first-order upwind scheme, which takes into account the curvature effect of the distribution curve of physical quantities between nodes and the influence of flow direction. It has high calculation accuracy and can adapt to complex fluid flow problems. The QUICK scheme is a method to improve the truncation of discrete equations. It is often used in hexahedral meshes. The QUICK scheme for convection terms has third-order accuracy and has high calculation accuracy. It is especially suitable for the problem of strong rotating flow and can reduce the degree of false expansion. By comparing the above three commonly used discrete schemes, combined with the fact that most meshes in this study are hexahedral meshes, and considering the rotating motion of blades, the QUICK scheme is adopted. A quick-type scheme is based on a weighted average of secondorder upwind and central interpolations of the variable. For the face $e$ in Figure 1, if the flow is from left to right, such a value can be written as

$$
\begin{aligned}
\varnothing e= & \theta\left[\frac{S_{d}}{S_{c}+S_{d}} \varnothing P+\frac{S_{c}}{S_{c}+S_{d}} \varnothing E\right] \\
& +(1-\theta)\left[\frac{S_{u}+2 S_{c}}{S_{u}+S_{c}} \varnothing P+\frac{S_{c}}{S_{u}+S_{c}} \varnothing W\right] .
\end{aligned}
$$

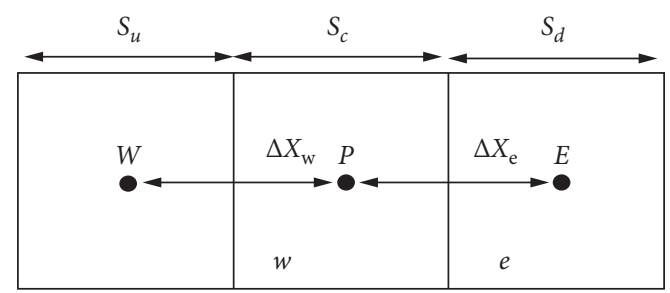

FIgURE 1: One-dimensional control volume.

TABle 1: Simulation test model parameters.

\begin{tabular}{llllll}
\hline$C_{1 \varepsilon}$ & $C_{2 \varepsilon}$ & $C_{3 \varepsilon}$ & $\sigma_{k}$ & $\sigma_{\varepsilon}$ & $C_{u}$ \\
\hline 1.44 & 1.92 & 0.09 & 1.0 & 1.3 & 0.09 \\
\hline
\end{tabular}

$\theta=1$ in the above equation results in a central secondorder interpolation while $\theta=0$ yields a second-order upwind value. The traditional QUICK scheme is obtained by setting $\theta=1 / 8$.

2.2. Turbulence Model. To take into consideration the effect of turbulence fluctuation on the flow inside the mill, a standard $k-\varepsilon$ model is adopted to calculate the velocity field of the gas phase. Furthermore, the RNG $k-\varepsilon$ model has been proven to be an appropriate model to describe the turbulence of industrial classifier flow [21]. The turbulent kinetic energy $(k)$ equation and fluid turbulent energy dissipation rate $(\varepsilon)$ equation are

$$
\begin{aligned}
\frac{\partial(\rho u)}{\partial t}+\frac{\partial\left(\rho k u_{i}\right)}{\partial x_{i}} & =\frac{\partial}{\partial x_{j}}\left[\left(u+\frac{u_{t}}{\sigma_{k}}\right)\right] \frac{\partial k}{\partial x_{j}}+G_{k}+G_{b}-\rho \varepsilon-Y_{M}+S_{k} \\
\frac{\partial(\rho \varepsilon)}{\partial t}+\frac{\partial\left(\rho \varepsilon u_{i}\right)}{\partial x_{i}} & =\frac{\partial}{\partial x_{j}}\left[\left(u+\frac{u_{t}}{\sigma_{\varepsilon}}\right)\right] \frac{\partial \varepsilon}{\partial x_{j}}+C_{1 \varepsilon} \frac{\varepsilon}{k}\left(G_{k}+C_{3 \varepsilon} G_{b}\right)-C_{2 \varepsilon} \rho \frac{\varepsilon^{2}}{k}+S_{\varepsilon} \\
G_{k} & =\mu_{t}\left(\frac{\partial u_{i}}{\partial x_{j}}+\frac{\partial u_{j}}{\partial x_{i}}\right) \frac{\partial u_{i}}{\partial x_{j}} \\
\mu_{t} & =\rho C_{u} \frac{k_{\varepsilon}^{2}}{\varepsilon}
\end{aligned}
$$

where $G_{k}$ and $G_{b}$ represent the generation of turbulence kinetic energy due to the mean velocity gradients and buoyancy, respectively. $Y_{M}$ is the effect of compressible turbulent pulsation expansion on the total dissipation rate. $\sigma_{k}$ and $\sigma_{\varepsilon}$ are Prandtl numbers corresponding to turbulent kinetic energy and dissipation rate, respectively. $S_{k}$ and $S_{\varepsilon}$ are user-defined source items. $\mu_{t}$ is the turbulent viscosity coefficient.

For incompressible fluids, user-defined source terms are not considered, and the constant values of $C_{1 \varepsilon}, C_{2 \varepsilon}, C_{3 \varepsilon}, \sigma_{k}$, $\sigma_{\varepsilon}$, and $C_{u}$ are listed in Table 1 .
2.3. Discrete Phase Model (DPM). According to the force balance acting on the particles, the trajectories of particles can be obtained from the equations of particle motion given by [22]

$$
\begin{aligned}
\frac{\mathrm{d} u_{p}}{\mathrm{~d} t} & =\frac{18 \mu}{\rho_{p} D_{p}^{2}} \frac{C_{D} \operatorname{Re}}{24}\left(u-u_{p}\right)+\frac{g\left(\rho_{p}-\rho\right)}{\rho_{p}}+F_{\text {other }}, \\
C_{D} & =a_{1}+\frac{a_{2}}{\operatorname{Re}}+\frac{a_{3}}{\operatorname{Re}^{2}} .
\end{aligned}
$$


Here, particles are supposed to be spherical. $a_{1}, a_{2}$, and $a_{3}$ are constants that apply to smooth spherical particles over several ranges of Re given by Morsi and Alexander [23], and the a's are defined as follows:

$$
a_{1}, a_{2}, a_{3}= \begin{cases}0,24,0, & 0<\operatorname{Re}<0.1, \\ 3.69,22.73,0.0903, & 0.1<\operatorname{Re}<1, \\ 1.222,29.1667,-3.8889, & 1<\operatorname{Re}<10, \\ 0.6167,46.5,-116.67, & 10<\operatorname{Re}<100, \\ 0.3644,98.33,-2778, & 100<\operatorname{Re}<1000, \\ 0.357,148.62,-47500, & 1000<\operatorname{Re}<5000, \\ 0.46,-490.546,578700, & 5000<\operatorname{Re}<10000, \\ 0.5191,-1662.5,5416700, & \operatorname{Re} \geq 10000,\end{cases}
$$

where $u$ and $u_{p}$ are the velocity of gas and particles, respectively. $\mu$ is the viscosity of the gas. $\rho$ and $\rho_{p}$ present the gas and particle density, respectively. Re is the Reynolds number. $D_{p}$ is the particle diameter. The drag coefficient is denoted by $C_{D}$. $F_{\text {other }}$ is other interaction forces. $g$ presents the gravitational acceleration. $a_{1}, a_{2}$, and $a_{3}$ are constants determined by the value of the Reynolds number Re.

In order to consider the dispersion effect of turbulence fluctuation on particles, Discrete Random Walk (DRW) is selected in fluent, and the interaction between particles and discrete vortices is considered. In the particle trajectory equation, the fluid velocity becomes instantaneous velocity:

$$
u=\bar{u}+u^{\prime}
$$

where $\bar{u}$ is the average velocity of the fluid and $u^{\prime}$ is the turbulent fluctuating velocity of the fluid.

According to the characteristics of particles passing through the turbulence, the fluctuating velocities $u^{\prime}, v^{\prime}$, and $w^{\prime}$ satisfying the Gaussian probability density distribution function are as follows:

$$
\begin{aligned}
u^{\prime} & =\zeta \sqrt{\left(u^{\prime}\right)^{2}}, \\
v^{\prime} & =\zeta \sqrt{\left(v^{\prime}\right)^{2}} \\
w^{\prime} & =\zeta \sqrt{\left(w^{\prime}\right)^{2}} \\
\sqrt{\left(u^{\prime}\right)^{2}} & =\sqrt{\left(v^{\prime}\right)^{2}}=\sqrt{\left(w^{\prime}\right)^{2}}=\sqrt{\frac{2 k}{3}}
\end{aligned}
$$

where $\zeta$ is a normally distributed random number, between -1 and 1.

2.4. Two-Phase Coupling. The finite volume method is used to solve the fluid governing equations. The pressure-velocity problem is solved by the classic SIMPLEC algorithm. The pressure difference compensation scheme uses the PRESTO! The QUICK scheme with good convergence is used for momentum, turbulent kinetic energy, and turbulent dissipation rate. In the calculation of two-phase flow, because the volume fraction is far less than $10 \%$, only the effect of gas phase on particle phase is considered. The control equations of the discrete phase and continuous phase are solved alternately until they both converge and finally realize bidirectional coupling calculation. The two-phase coupling effect only considers the momentum exchange, and the mass and heat exchange can be ignored. The particle momentum equation is as follows:

$$
F=\sum\left[\frac{18 \mu C_{D} \operatorname{Re}}{\rho_{p} d_{p}^{2} 24}\left(u_{p}-u\right)+F_{\text {other }}\right] \dot{m}_{p} t
$$

where $F$ presents the particle momentum and $\dot{m}_{p}$ is the mass flow rate. $t$ is the time step. The other parameters have been defined in equation (6).

Note that only momentum exchange is considered in this work and it is underrelaxed, so that

$$
F_{\text {new }}=F_{\text {old }}+\alpha\left(F_{\text {calculated }}-F_{\text {old }}\right),
$$

where $\alpha$ is the underrelaxation factor for particles/gas that you can set in the Solution Controls panel. The default value for $\alpha$ is 0.5 . This value may be reduced to improve the stability of coupled calculations. Note that the value of $\alpha$ does not influence the predictions obtained in the final converged solution.

2.5. Calculation Model of Rotating Blade Region. The simulation of the motion region mainly includes the single reference frame model (SRF), multiple reference frame model (MRF), sliding mesh model, and dynamic mesh model. In the numerical calculation of the flow field of the vertical roller mill, in order to ensure the calculation accuracy, the global mesh size of the blade region is set as 0.01 , and the number of meshes is 6.17 million. With such a number of meshes, the calculation efficiency of the sliding mesh model and the dynamic mesh model is very low, and the numerical simulation work cannot be solved. SRF model cannot be used to solve the complex flow field model of the vertical roller mill. Therefore, the MRF model is selected to simulate the rotating process of the blade region, and the blade rotation process is approximated as steady motion. In addition, the MRF model fits well with the selected pressure difference compensation scheme (PRESTO!). The calculation domain of the vertical roller mill is divided into multiple small subdomains. The governing equations of the flow field are solved in each subdomain. The static region is controlled by the continuity and momentum equations, while the rotating region is controlled by the equations of the moving reference frame. The velocity is transformed into absolute velocity at the interface to exchange the flow field information. Figure 2 shows the coordinate system for relative velocity.

The position vector relative to the origin of the zone rotation axis is defined as

$$
\vec{r}=\vec{x}-\vec{x}_{o}
$$




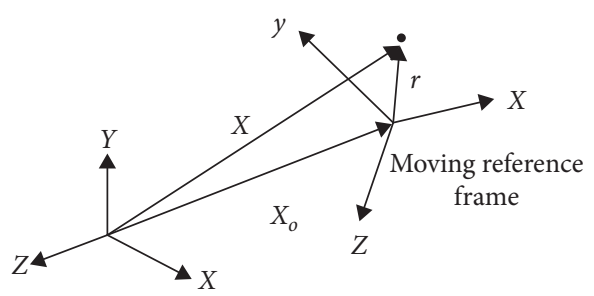

Absolute reference frame

FIgURE 2: Coordinate system for relative velocity.

where $\vec{x}$ is the position in absolute Cartesian coordinates and $\vec{x}_{o}$ is the origin of the zone rotation axis, as shown in Figure 1.

The relative velocity in the moving reference frame can be converted to the absolute (stationary) frame of reference using the following equation:

$$
\vec{v}=\vec{v}_{r}+(\vec{\omega} \times \vec{r})+\vec{v}_{t},
$$

where $\vec{v}$ is the velocity in the absolute inertial reference frame, $\vec{v}_{r}$ is the velocity in the relative noninertial reference frame, and $\vec{v}_{t}$ is the translational velocity of the noninertial reference frame.

Using this definition of absolute velocity, the gradient of the absolute velocity vector is given by

$$
\nabla \vec{v}=\nabla \vec{v}_{r}+\nabla(\vec{\omega} \times \vec{r})
$$

2.6. Geometry Model and Boundary Conditions. An industryemployed vertical roller mill of a $10 \mathrm{t} / \mathrm{h}$ (ton per hour) pyrophyllite production line in Tongxiang city (Jushi Group Co., Ltd., Zhejiang Province, China) is chosen to be the simulation object. It has 2 rollers and the diameter of the millstone is $2400 \mathrm{~mm}$. The 3D model is shown in Figure 3.

The number of meshes affects the calculation speed and accuracy of the numerical simulation directly. The ANSYS ICEM CFD package is used to mesh the vertical roller mill flow field model and divide it into nine domains in this work. The MRF (multiple reference frames) multicoordinate system method is applied to the blade mesh. In order to ensure the mesh-independence of the calculation results, this article changes the number of meshes by changing the global mesh and divides them into five groups of different numbers of meshes. The influence of the mesh number on the result has been measured by comparing the wind speed at the wind ring. The results are shown in Table 2 . When the number of meshed exceeds 5.34 million, the wind speed at the wind ring has no significant difference. Considering the calculation time and accuracy, the 6.17 million mesh model is selected for numerical simulation and presented in Figure 4.

Table 3 lists the actual operating condition parameters and Table 4 presents the content of each particle size interval of input particles. To improve the accuracy of calculation, the simulation is carried out according to the practical distribution of particles. To ensure the accuracy of the particle size distribution of the flow field simulation, the

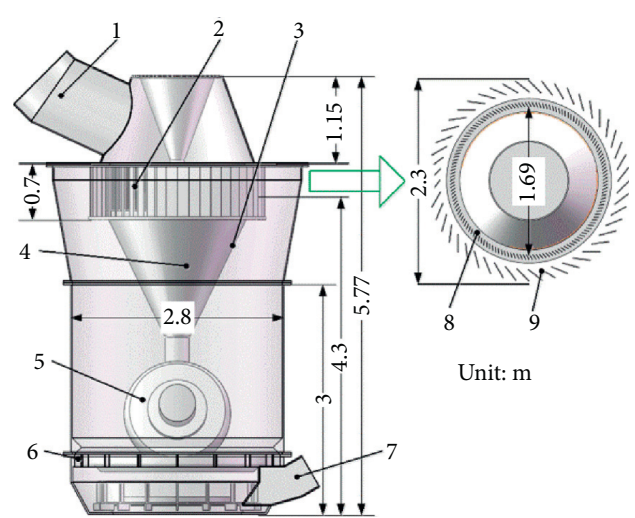

Figure 3: The 3D geometry model of the vertical roller mill. 1: separator outlet; 2: separator; 3: shell; 4: return hopper; 5: grinding roller; 6: wind ring; 7: air inlet; 8: blade; 9: guide vane.

TABLE 2: The effect of the number of meshes on the speed at the wind ring.

\begin{tabular}{llllll}
\hline Number of meshes (million) & 3.23 & 4.86 & 5.34 & 6.17 & 7.25 \\
Wind ring velocity (m/s) & 30.4 & 40.5 & 50.8 & 51.2 & 51.3 \\
\hline
\end{tabular}

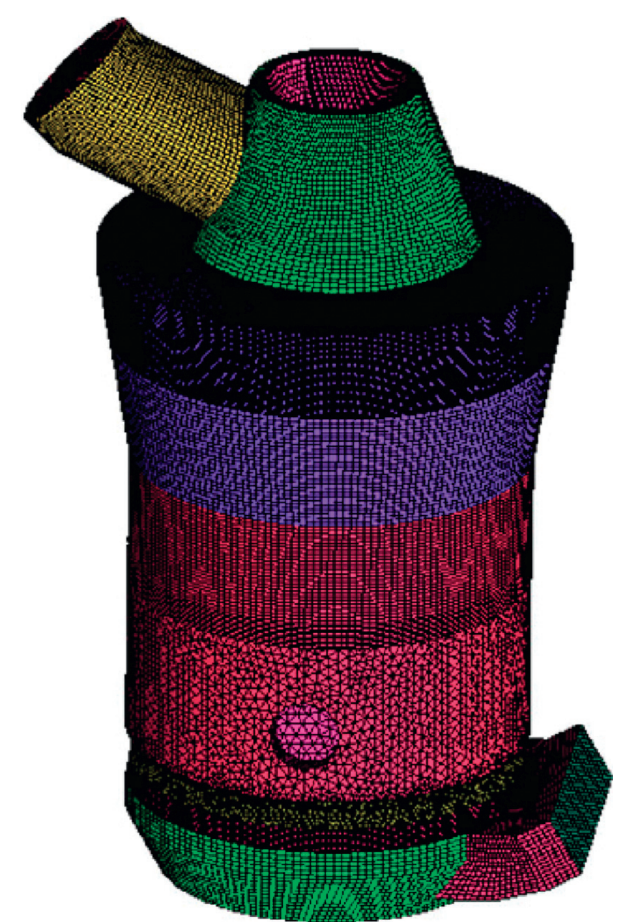

Figure 4: 3D mesh of vertical roller mill.

TABLE 3: Boundary conditions of the flow field in the vertical mill.

\begin{tabular}{lcc}
\hline Boundary name & Unit & Results \\
\hline Inlet wind speed & $\mathrm{m} / \mathrm{s}$ & 18.2 \\
Inlet pressure & $\mathrm{Pa}$ & 0 \\
Outlet pressure & $\mathrm{Pa}$ & -6500 \\
Particle density & $\mathrm{t} / \mathrm{m}^{3}$ & 1.5 \\
Blade speed & $\mathrm{r} / \mathrm{min}$ & 200 \\
Feed volume & $\mathrm{kg} / \mathrm{s}$ & 3.3 \\
Particle velocity & $\mathrm{m} / \mathrm{s}$ & 4.76 \\
\hline
\end{tabular}


TABLE 4: Content of each particle size interval.

\begin{tabular}{lcccccccccc}
\hline Particle size interval $(\mu \mathrm{m})$ & $0-10$ & $10-20$ & $20-30$ & $30-40$ & $40-50$ & $50-60$ & $60-70$ & $70-80$ & $80-90$ & $90-100$ \\
Content $(\%)$ & 19.5 & 25 & 20 & 15.5 & 12 & 5 & 1 & 1 & 0.5 & 0.5 \\
\hline
\end{tabular}

particles at the edge of the millstone and the position above the wind ring in actual production are sampled, and the particle size of samples was analyzed by BT-2001 laser distributor. The obtained particles were divided into 10 groups as input particles. The average particle size and distribution coefficient are $63 \times 10^{-6} \mathrm{~m}$ and 1.76, respectively.

\section{Results and Discussion}

\subsection{Flow Field Description}

3.1.1. Velocity Field. Figure 5 shows the cloud diagram of the vertical roller mill with different cross-sectional speeds $(\mathrm{m} / \mathrm{s})$ and local velocity vector. It can be seen that the wind speed at the wind ring is the largest in the entire basin with a maximum airflow speed of $51.1 \mathrm{~m} / \mathrm{s}$, and the wind speed of the wind ring has a direct impact on the classification efficiency. Figure 6 presents the locally enlarged view of $Z=0$ section velocity vector. It can be obtained that there is an obvious vortex phenomenon between the wind ring and the grinding roller, and this phenomenon is consistent with what Wang [9] and Sun [10] described. The appearance of the vortex will make the particles fully contact with the air, which is conducive to the full coupling of the particles and the airflow. However, the vortex will increase the turbulent kinetic energy and turbulent dissipation rate, resulting in a decrease in airflow velocity and an increase in energy loss.

3.1.2. Pressure Field. The internal pressure difference of the vertical roller mill is a very significant parameter for studying the flow field. The stable pressure difference means that the feed amount and the output are balanced. When the pressure difference rises, it means that the feeding amount is greater than the output, and the internal circulation rate, wind resistance, and energy consumption of the system will increase. The pressure nephogram of $Z=0$ section is shown in Figure 7. The average pressures of each region are presented in Table 5 . It can be seen that the maximum flow field pressure of the vertical roller mill is at the air inlet. The pressure difference of the whole machine is equivalent to the pressure difference between separator out and the air inlet is about $4575 \mathrm{~Pa}$. The pressure difference of the separator is equivalent to the pressure difference between separator outlet and separator inlet which is about $1481 \mathrm{~Pa}$.

3.1.3. Discrete Phase Analysis. Figure 8 presents the particle streamline diagram of the internal flow field of the vertical roller mill. The trajectory in the figure represents the trajectory of particles, and the numerical value means the residence time of particles in the vertical mill. It can be indicated that most of the particles below $45 \mu \mathrm{m}$ are selected by the separator after $3.7 \mathrm{~s}$. However, due to the vortex inside the vertical roller mill, some particles are selected by the separator after about $16 \mathrm{~s}$. It can also be seen that the vortex in the vertical mill has a great influence on the particle trajectory, resulting in the increase of internal circulation and energy consumption.

3.1.4. Classification Efficiency Analysis. Table 6 shows the classification efficiency of each particle size by numerical simulation. It can be obtained that the classification efficiency of particles smaller than $45 \mu \mathrm{m}$ is above $94.59 \%$, while particles larger than $89 \mu \mathrm{m}$ cannot be selected. The results show that the mass flow rate of sieve residue is $2.5 \mathrm{~kg} / \mathrm{s}$ and the total mass flow rate of the outlet is $2.71 \mathrm{~kg} / \mathrm{s}$. The classification efficiency is defined as the percentage of particles at the separator outlet and the particles entering the separator. Therefore, the classification efficiency of outlet particle size below $45 \mu \mathrm{m}$ is $92 \%$.

3.1.5. Model Validation. In order to verify the numerical model described before, the simulated results have been compared with the practical running data. The particle size distributions at the outlet of the vertical roller mill are focused. Figure 9 shows practical particle size distribution. Table 7 presents a comparison between the simulation results and the plant data. It could be found that they show the same trend and the maximum error is $6.7 \%$. Moreover, the comparison of pressure simulation results with plant data is shown in Table 8, and the maximum error is $2.14 \%$. Therefore, it can be concluded that the adopted numerical model in this work is correct and reasonable at least.

\subsection{Flow Field and Classification Efficiency Analysis}

3.2.1. Effect of Blade Structure. There are four types of blades shown in Figure 10. For the convenience of analysis, the four kinds of blade shapes are distinguished by Type I, Type II, Type III, and Type IV, respectively.

The local velocity vector diagram with four blade shapes is shown in Figure 11. It can be seen that the velocity and flow direction of the four blades are different. Compared with Type I, the flow direction and distribution of Type II are almost the same. Due to the corner at the near center of Type II, the velocity direction deflects along the bend angle after the collision between the flow and the angle, resulting in some particles unable to enter the cage. Compared with Type I, due to the existence of a bend angle in Type III, the speed direction is deflected along the angle to produce a rotation, which prevents the particles from smoothly entering the rotating cage. Compared with Type I, Type IV has a greater radial velocity in the air flow in the classification zone, which is beneficial to the particles entering the cage. It can be indicated that the flow field of Type I is the most stable, while that of Type III is the most unstable. Figure 12 presents the 


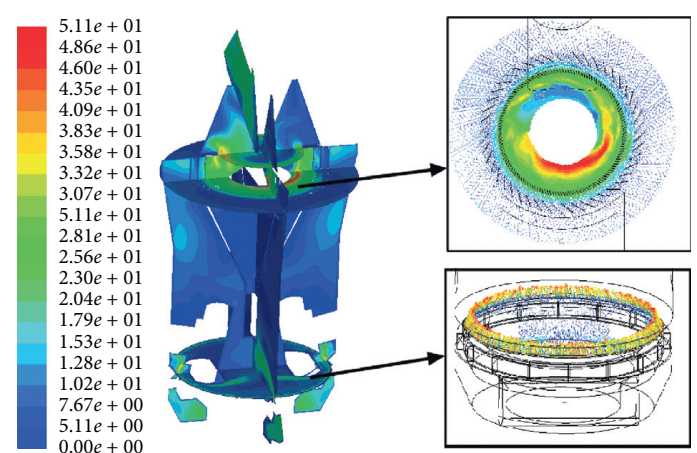

Figure 5: Cloud diagram of the vertical roller mill with different cross-sectional speeds $(\mathrm{m} / \mathrm{s})$ and local velocity vector.

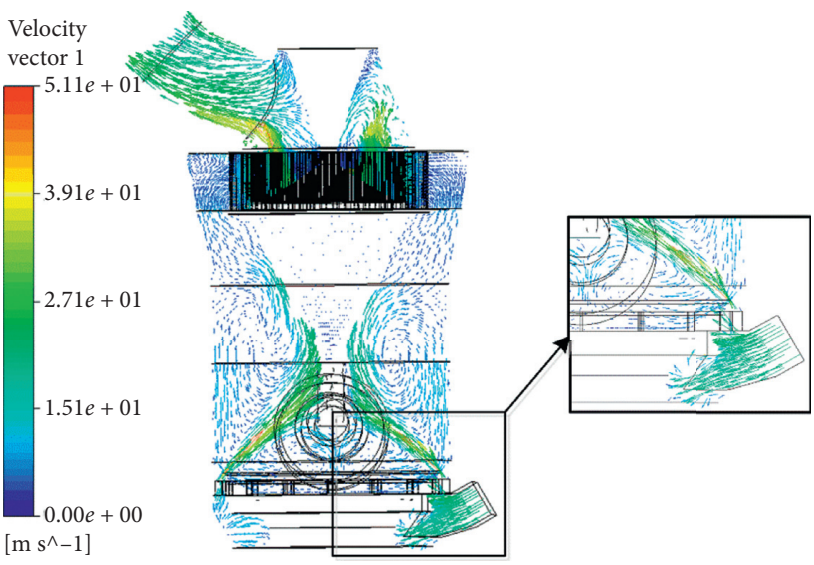

Figure 6: Locally enlarged view of $Z=0$ section velocity vector.

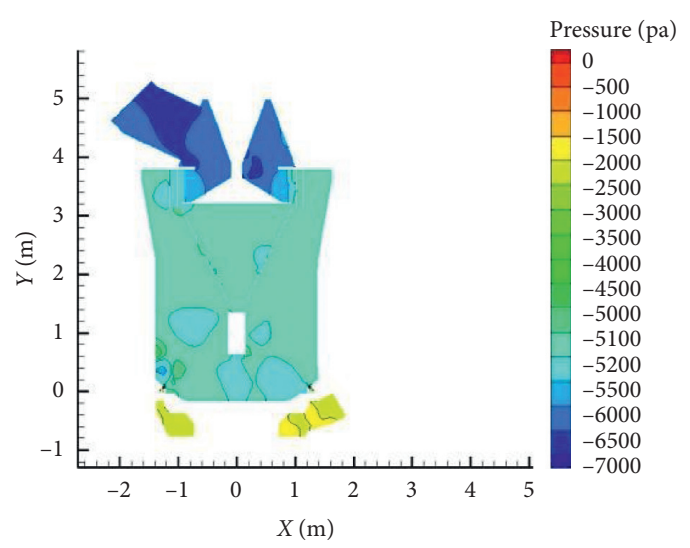

Figure 7: Pressure nephogram of $Z=0$ section.

speed variation chart of Line 1 with four blade shapes. It can be obtained that the overall trend of velocity from P1 to P2 increases. The inlet angle of the Type III blade blocks the airflow, which results in a decrease of airflow velocity. The velocity of Type II and Type IV is reduced due to the existence of the angle near the center of the blade.

Figure 13 shows the differential pressure and classification efficiency of different shapes of blades. It can be seen that Type IV has a significant effect on the pressure difference compared with other types. The pressure differences of the separator and whole machine are $1217 \mathrm{~Pa}$ and $4325 \mathrm{~Pa}$, respectively, while Type I has the highest pressure difference, and the corresponding results are $1481 \mathrm{~Pa}$ and $4575 \mathrm{~Pa}$, respectively. It can be indicated that Type IV has obvious advantages of low resistance. It can effectively reduce the resistance and energy consumption inside the mill by selecting Type IV. It can be obtained that the order of classification efficiency from high to low is Type IV > Type II > Type I > Type III as the particle size is less than $45 \mu \mathrm{m}$. However, when the particle size is less than $80 \mu \mathrm{m}$, the order is Type III > Type I > Type IV > Type II. The factory usually takes $45 \mu \mathrm{m}$ particle size as the production index. The more content below $45 \mu \mathrm{m}$ indicates higher product quality. It can also be indicated that compared with the traditional Type I, Type II and Type IV are beneficial to the entrance of fine powder and the throwing out of coarse powder, which helps reduce the local vortex between the blades and improve the classification efficiency.

3.2.2. Effect of Blade Number. Figures 14 and 15 show the local velocity vector diagram and velocity nephogram of different numbers of blades. It is concluded that regardless of the number of blades, the airflow is very uniform in the classification area, and the airflow distribution in the near center region of the blade is more uniform. This is due to the increasing number of blades and because the effect of the blades on the airflow is also enhanced, and the vortex between the blades continues to decrease.

The number of blades has a great influence on the flow field and classification efficiency. Combined with actual production experience, the number of blades is selected to be $80,96,125,144$, and 150 for numerical analysis. Figure 16 presents the differential pressure and classification efficiency of different numbers of blades. It can be seen that the pressure difference of the separator and whole machine changes very little as the number of blades is 80,96 , and 125 . However, when the blade number reaches 144 and 150, the pressure difference increases sharply. This phenomenon indicates that when the number of blades is in a certain range, the gas-solid two-phase flow into the cage is not affected by the blade spacing. However, the probability of collision between the particles and the blades increases as the number increases, resulting in an increase in the cycle load 
TABle 5: Average pressure of each region.

\begin{tabular}{lccccc}
\hline Region & Air inlet & Separator outlet & Separator inlet & Top of wind ring & Bottom of wind ring \\
\hline Pressure $(\mathrm{Pa})$ & -1926 & -6500 & -5020 & -2447 & -2277 \\
\hline
\end{tabular}

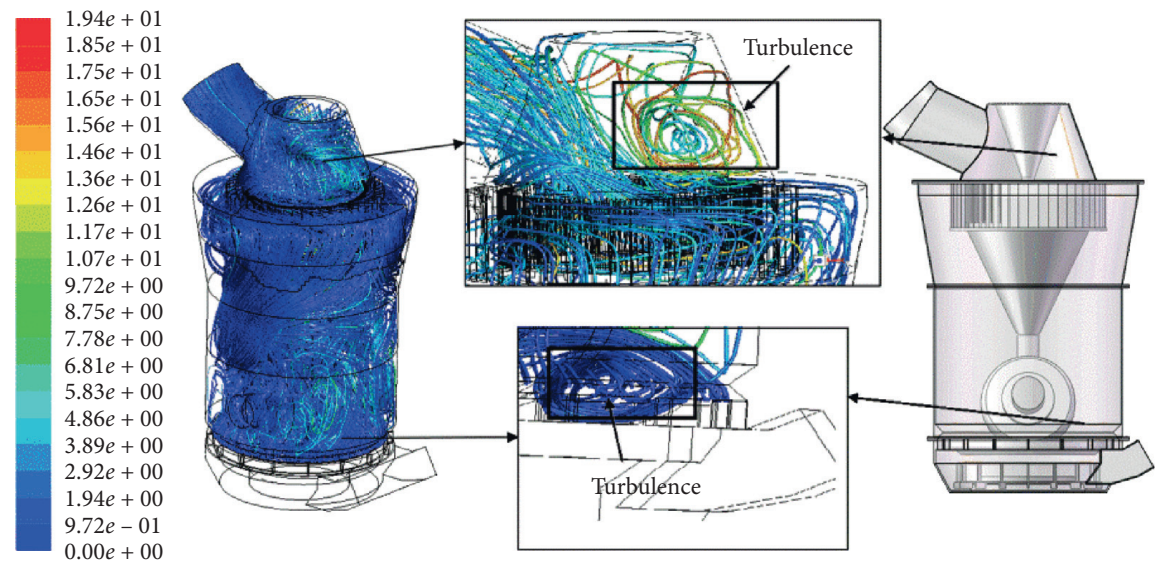

Figure 8: Particle streamline diagram of the internal flow field of vertical roller mill.

TABLE 6: Classification efficiency of each particle size by numerical simulation.

\begin{tabular}{lcccccccccc}
\hline Particle size $(\mu \mathrm{m})$ & $0-10$ & $10-20$ & $20-30$ & $30-40$ & $40-50$ & $50-60$ & $60-70$ & $70-80$ & $80-90$ & $90-100$ \\
Inlet mass flow $(\mathrm{kg} / \mathrm{s})$ & 0.501 & 0.707 & 0.549 & 0.424 & 0.327 & 0.251 & 0.192 & 0.148 & 0.114 & 0.087 \\
Outlet mass flow $(\mathrm{kg} / \mathrm{s})$ & 0.5 & 0.703 & 0.546 & 0.421 & 0.324 & 0.134 & 0.073 & 0.001 & 0 & 0 \\
Classification efficiency $(\%)$ & 99.9 & 99.5 & 99.4 & 99.4 & 99.2 & 53.36 & 38.01 & 6.52 & 0 & 0 \\
\hline
\end{tabular}

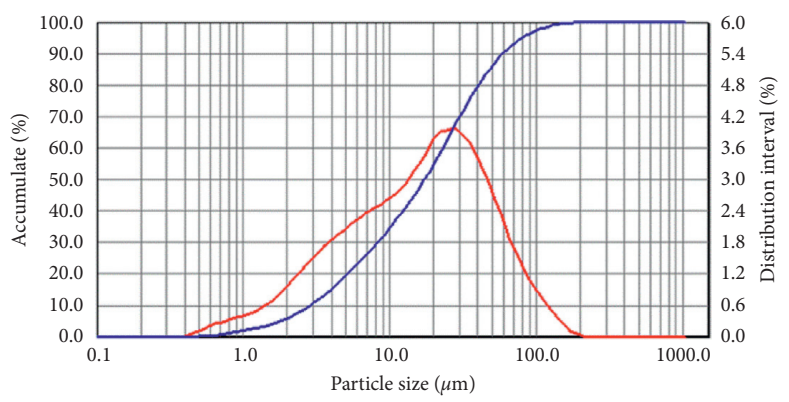

Figure 9: Particle size analysis report of pyrophyllite products. The red line represents the size distribution interval, and the blue line represents the cumulative amount.

TABLE 7: Comparison between simulation results of particle size distribution and plant data.

\begin{tabular}{|c|c|c|c|c|c|c|c|c|c|c|}
\hline Size $(\mu \mathrm{m})$ & $0-10$ & $10-20$ & $20-30$ & $30-40$ & $40-50$ & $50-60$ & $60-70$ & $70-80$ & $80-90$ & $90-100$ \\
\hline Simulation results (cumulants \%) & 18.5 & 44.5 & 64.7 & 80.3 & 92.3 & 97.2 & 99.9 & 100 & 100 & 100 \\
\hline Plant data (cumulants \%) & 18.1 & 41.5 & 62.2 & 80 & 93.5 & 97.2 & 98.9 & 100 & 100 & 100 \\
\hline Error $(\%)$ & 2.1 & 6.7 & 3.86 & 0.37 & 1.28 & 0 & 1 & 0 & 0 & 0 \\
\hline
\end{tabular}

TABLE 8: Comparison of pressure simulation results with plant data.

\begin{tabular}{lccc}
\hline Parameter & Air inlet & Separator outlet & Differential pressure \\
\hline Simulation results $(\mathrm{Pa})$ & -1926 & -6500 & 4574 \\
Plant data $(\mathrm{Pa})$ & -1900 & -6575 & 4675 \\
Error $(\%)$ & 1.3 & 1.14 & 2.14 \\
\hline
\end{tabular}




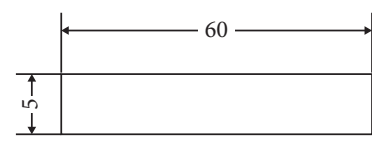

(a)

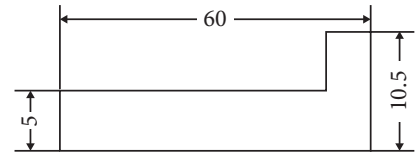

(b)

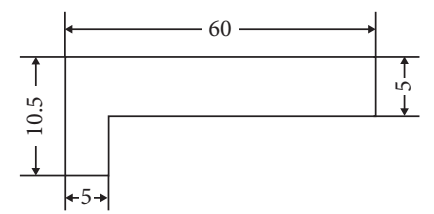

(c)

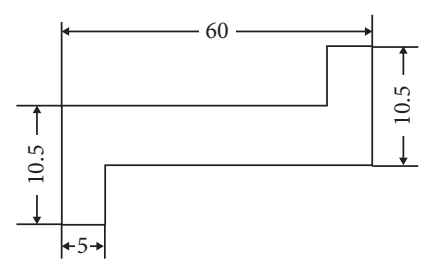

(d)

Figure 10: Four types of blade shapes. (a) Type I. (b) Type II. (c) Type III. (d) Type IV.

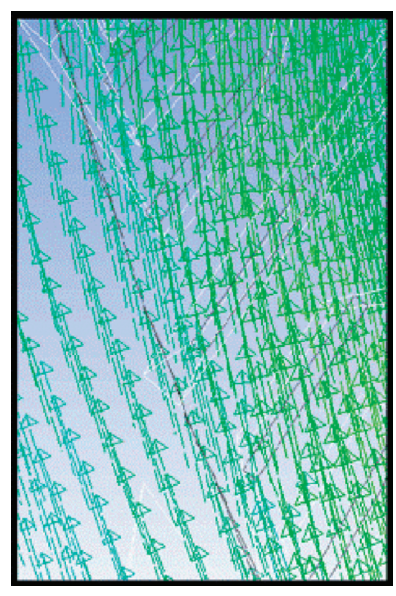

(a)

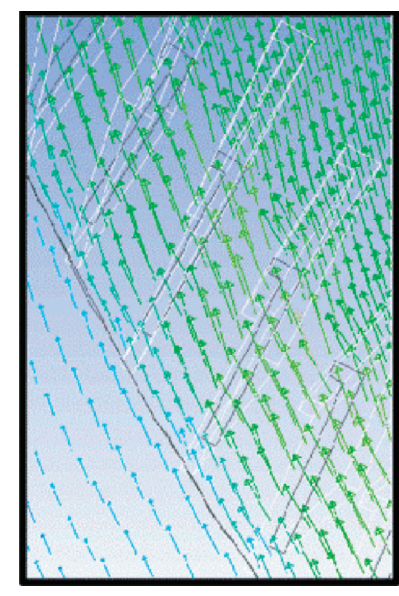

(b)

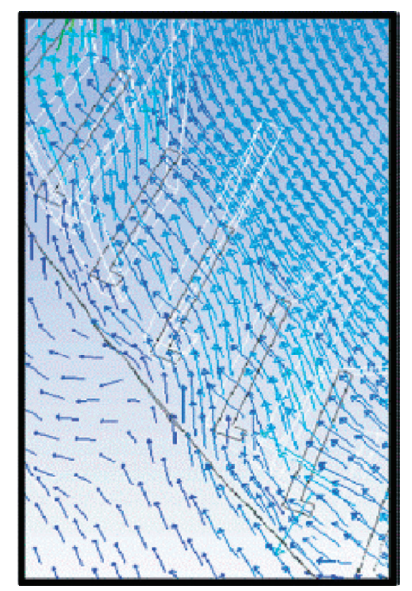

(c)

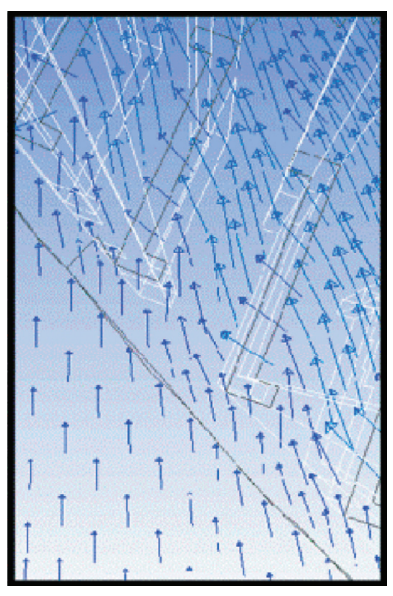

(d)

Figure 11: Local velocity vector diagram with four blade shapes. The blade rotation speed and number are $200 \mathrm{r} / \mathrm{min}$ and 144 , respectively. (a) Type I. (b) Type II. (c) Type III. (d) Type IV.

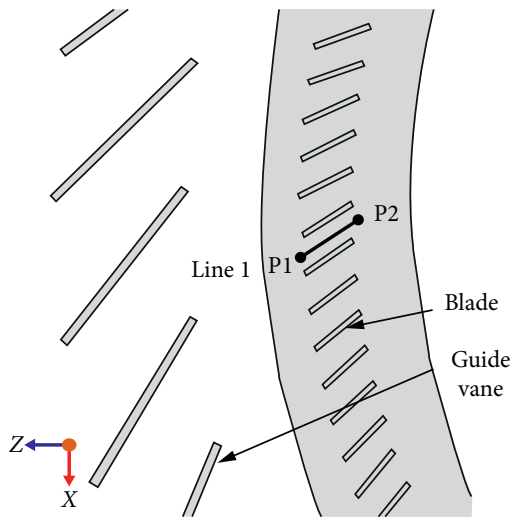

(a)

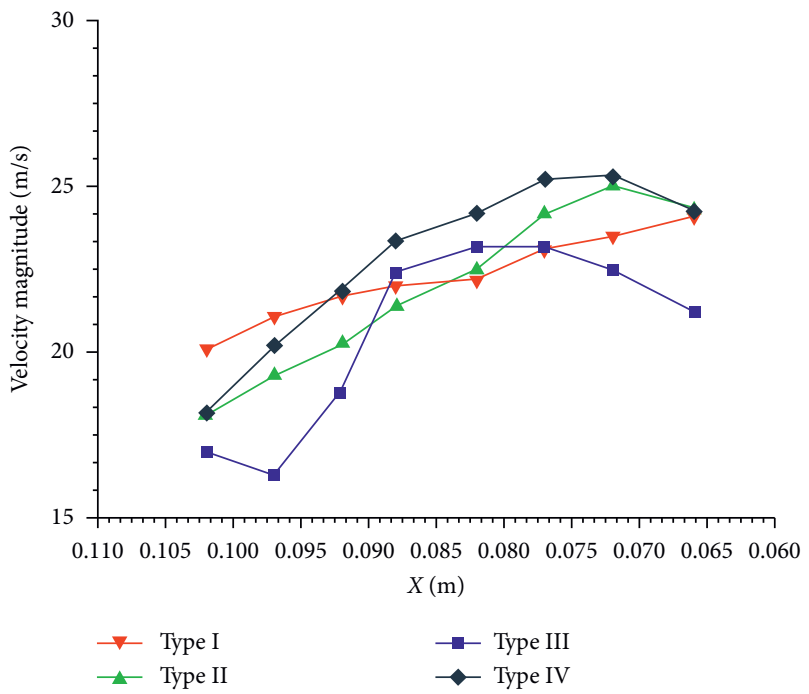

(b)

Figure 12: (a) The data collection of Line 1 established on the $Y=3.4 \mathrm{~m}$ section, with the starting point coordinates $\mathrm{P} 1(0.102,3.4,0.89)$ and the ending coordinates P2 $(0.066,3.4,0.84)$. (b) Speed variation chart of Line 1 with four blade shapes.

rate and the pressure difference between the separator and the whole machine. It can be inferred that the classification efficiency increases with the increasing number of blades as the particle size is less than $45 \mu \mathrm{m}$. However, the classification efficiency decreases with the increasing number of blades as the particle size is less than $80 \mu \mathrm{m}$. This phenomenon indicates that the number of blades has a great influence on the classification efficiency. When the number of blades is too small, it will cause a rough running phenomenon and increase the content of coarse particles. 


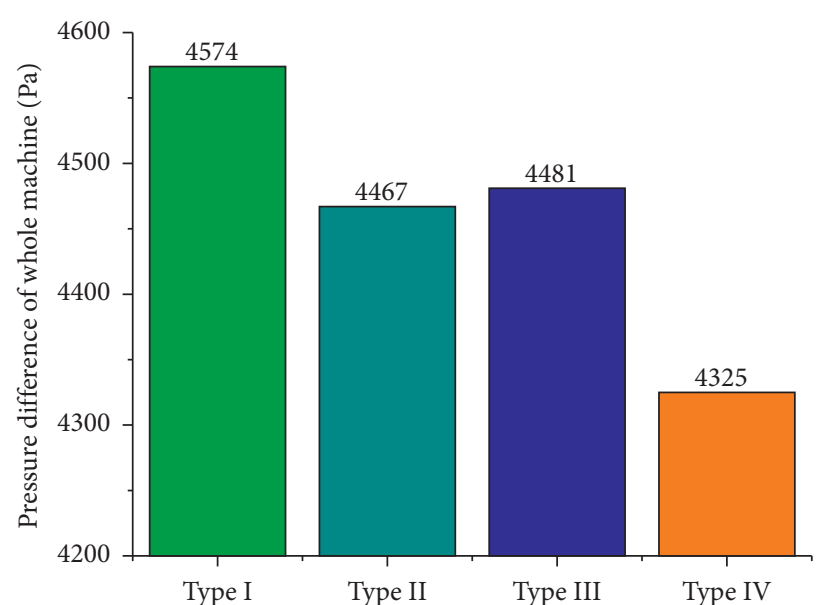

(a)

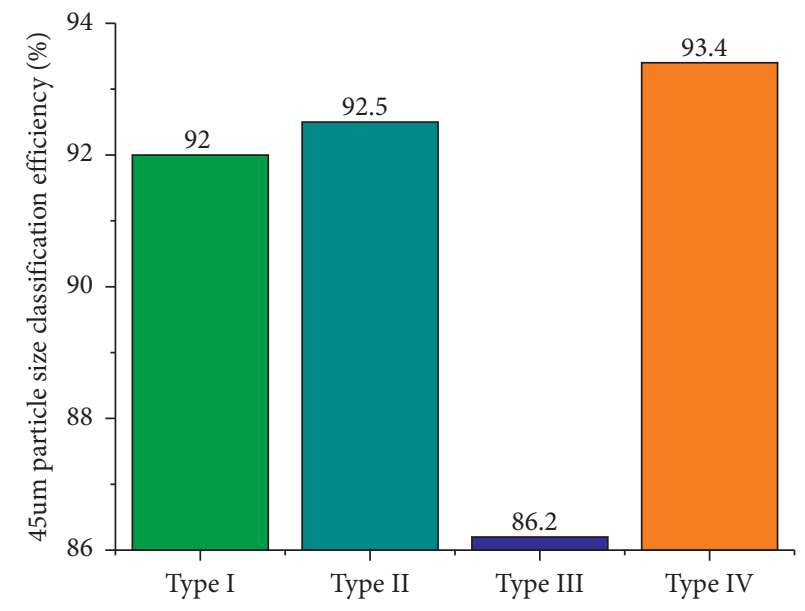

(c)

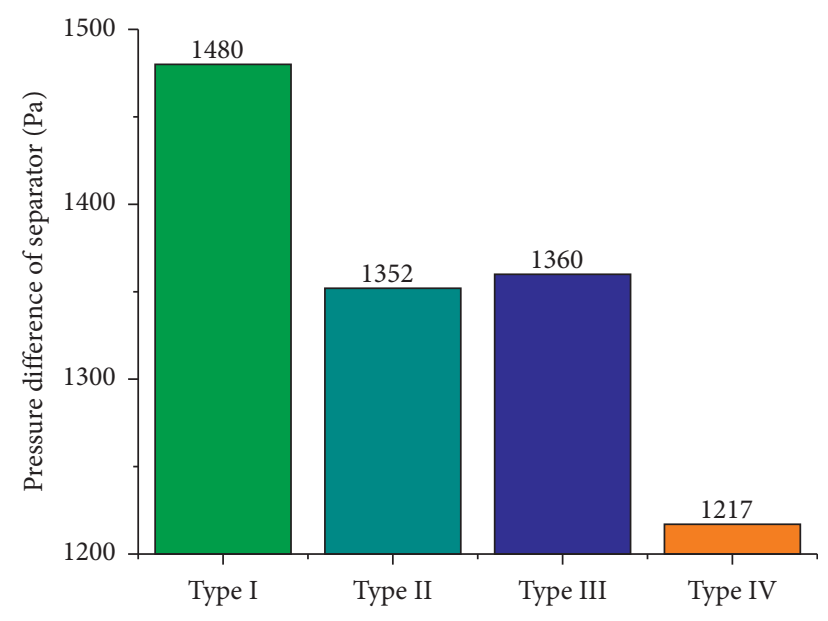

(b)

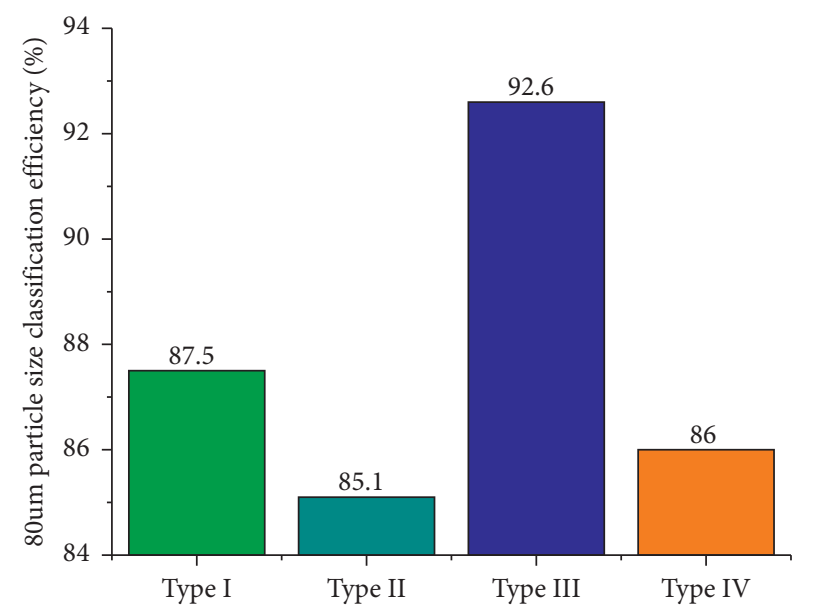

(d)

Figure 13: Relations between differential pressure, classification efficiency, and four different blades. (a) The pressure difference of the whole machine. (b) The pressure difference of separator. (c) The $45 \mu \mathrm{m}$ particle size classification efficiency. (d) The $80 \mu \mathrm{m}$ particle size classification efficiency. The results of the same type of blade analysis are shown in the same color.

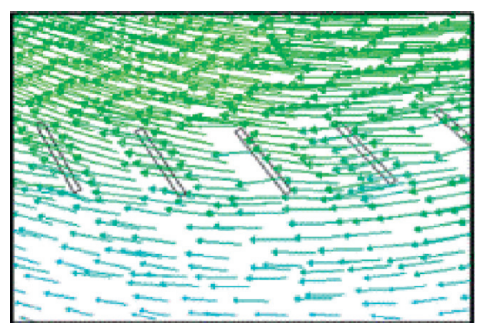

(a)

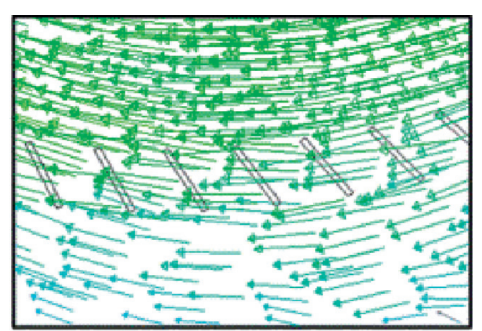

(b)

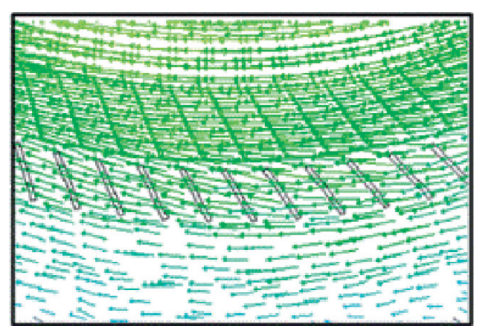

(c)

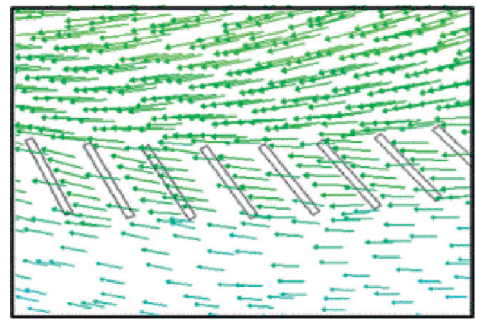

(d)

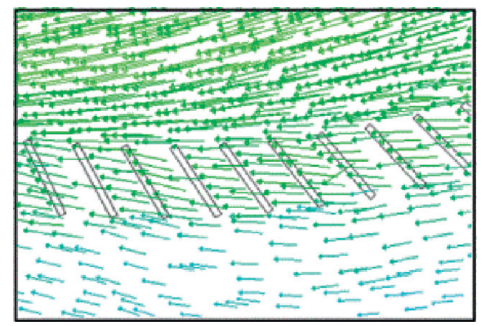

(e)

Figure 14: The local velocity vector maps with different numbers of blades. From (a) to (e), the quantity is $80,96,125,144$, and 150 , respectively. The blade rotation speed keeps at $200 \mathrm{r} / \mathrm{min}$ and the shape is Type I. 


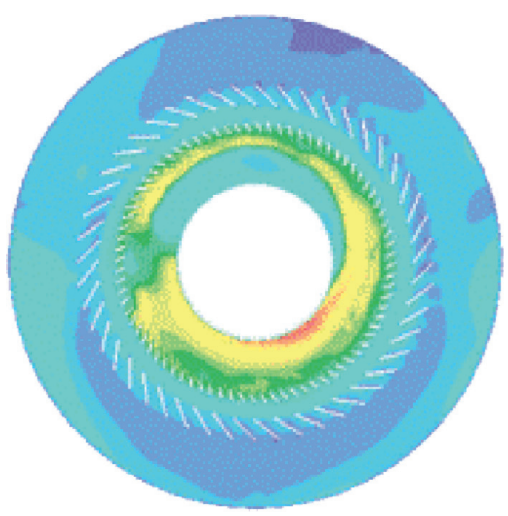

(a)

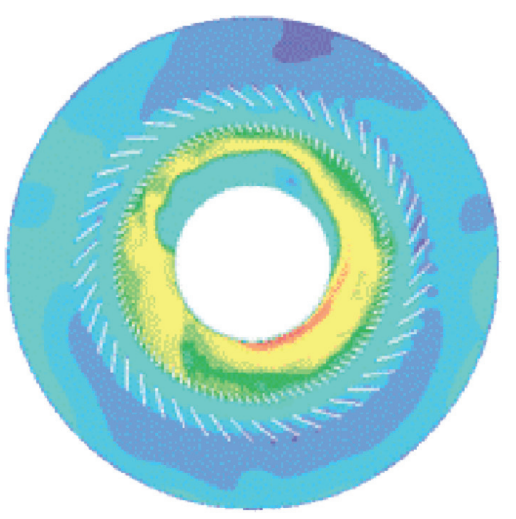

(b)

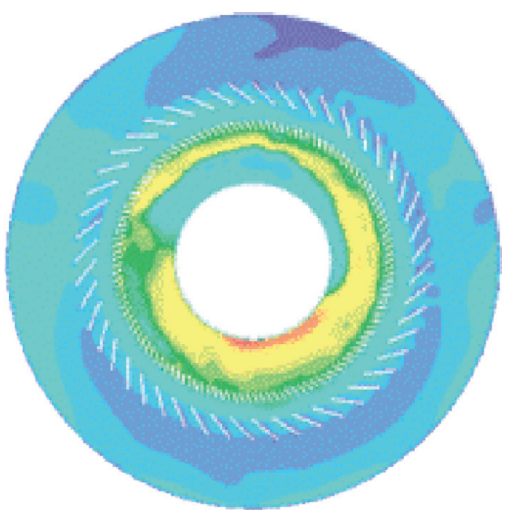

(c)

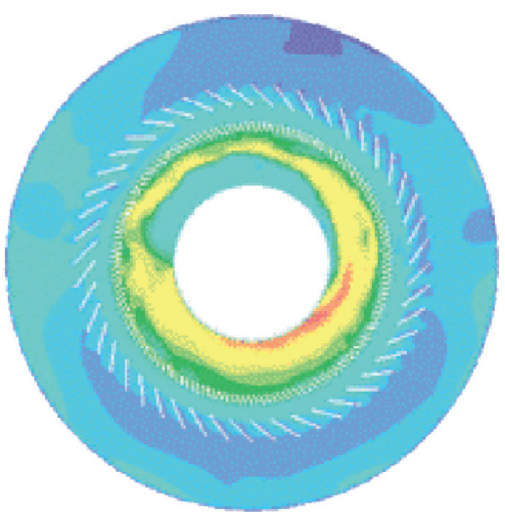

(d)

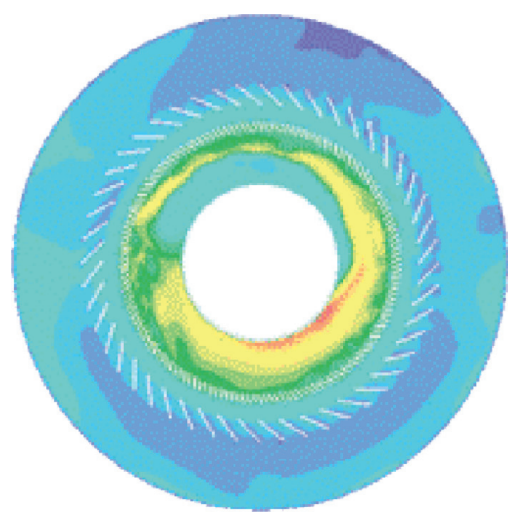

(e)

Figure 15: The local velocity nephogram with different numbers of blades. From (a) to (e), the quantity is $80,96,125,144$, and 150 , respectively.

However, when there are too many blades, qualified products cannot be screened out in time, which increases the internal resistance and energy consumption. Selecting a reasonable number of blades can improve the classification and screening performance of the vertical roller mill. Considering the advantages of classification efficiency and low resistance, the number of blades can be selected to be 144 .

3.2.3. Effect of Blade Rotating Speed. Figure 17 shows the velocity nephogram of $Y=3.4 \mathrm{~m}$ section. The data collection of Line 2 and Line 3 is established on the $Y=3.4 \mathrm{~m}$ section: Line 2 with the starting point coordinates P3 $(-0.81,3.4,0)$ and the ending coordinates $\mathrm{P} 4(-0.51,3.4,0)$ and Line 3 with the starting point coordinates $\mathrm{P} 5(0.81,3.4,0)$ and the ending coordinates P6 $(0.51,3.4,0)$. Figure 18 presents the speed curve of Line 2 and Line 3 at different speeds. It is found that the velocity from P3 to P4 increases first and then decreases. There is a speed turning point in the middle, and then the air velocity gradually stabilizes. The reason for this phenomenon is that the space in this area increases sharply, and the airflow suddenly expands, which leads to a decrease in velocity. The airflow speed gradually stabilizes near the center of rotation because there is no influence of the separator outlet. It can also be seen that the velocity P5 to P6 increases suddenly and then decreases gradually, and the speed of Line 3 turning point is significantly higher than that of Line 2; the main reason is that there is no separator outlet on this side.

The blade rotating speed also has an important impact on the flow field and classification efficiency of the vertical roller mill. Figure 19 shows the differential pressure and classification efficiency of blades at different speeds. It can be seen that blade rotation speed has a great influence on the internal pressure difference and classification efficiency. The pressure difference between the whole machine and separator continues to increase as the $\mathrm{f}$ the blade rotating speed increases. This is because the collision between the blade and gas-solid two-phase flow is intensified as the rotating speed increases, resulting in large pressure difference and energy consumption. It can be obtained that the classification efficiency is proportional to the speed as the particle size is less than $45 \mu \mathrm{m}$. However, the classification efficiency is inversely proportional to the speed as the particle size is less than $80 \mu \mathrm{m}$. This phenomenon indicates that coarse particles are not easy to be separated in the process of increasing rotating speed, resulting in the fact that the classification efficiency continues to decrease. Therefore, controlling the reasonable blade rotating speed can improve the classification performance of the vertical roller mill. 


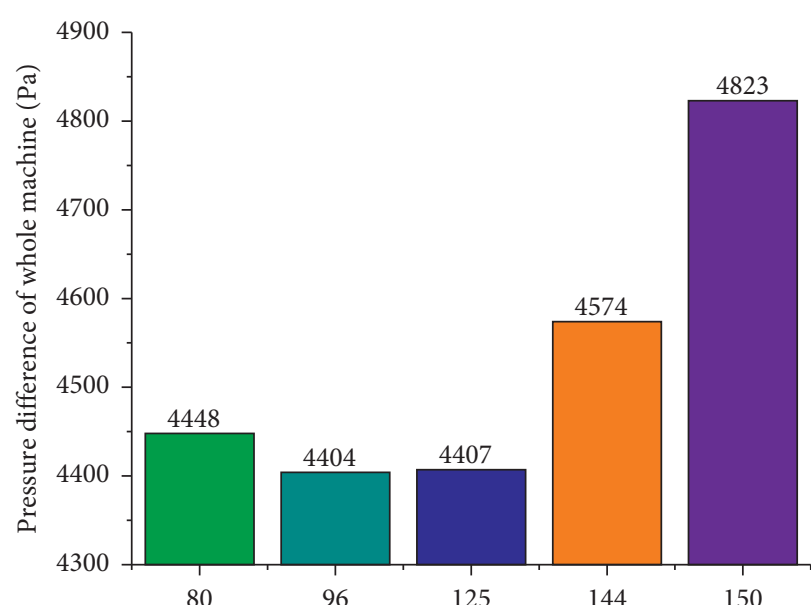

(a)

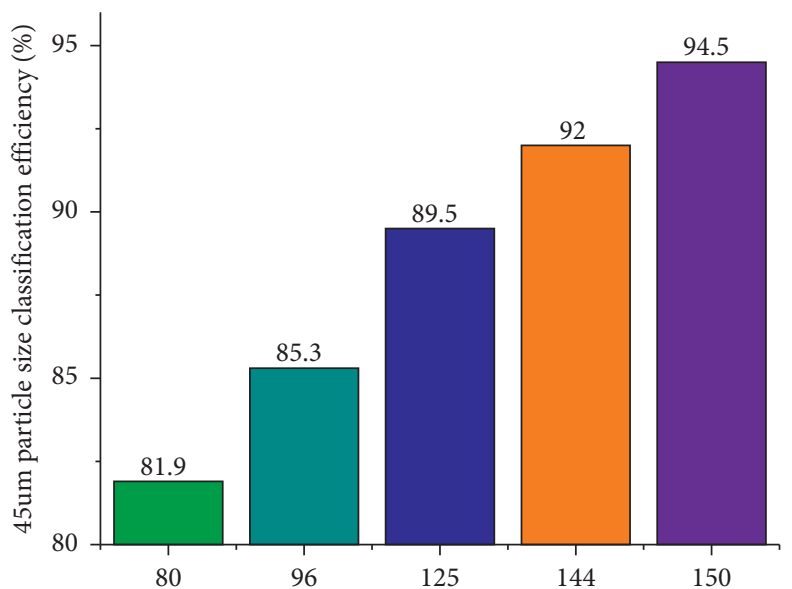

(c)

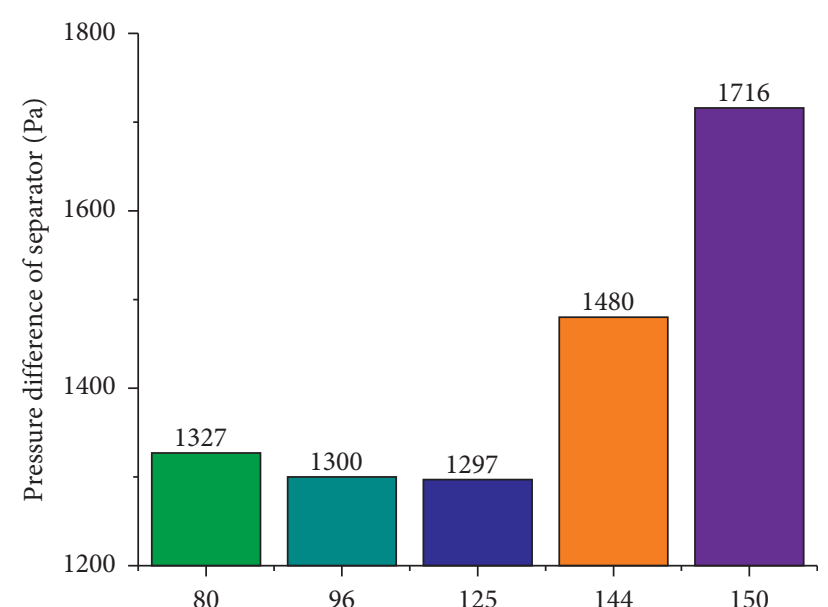

(b)

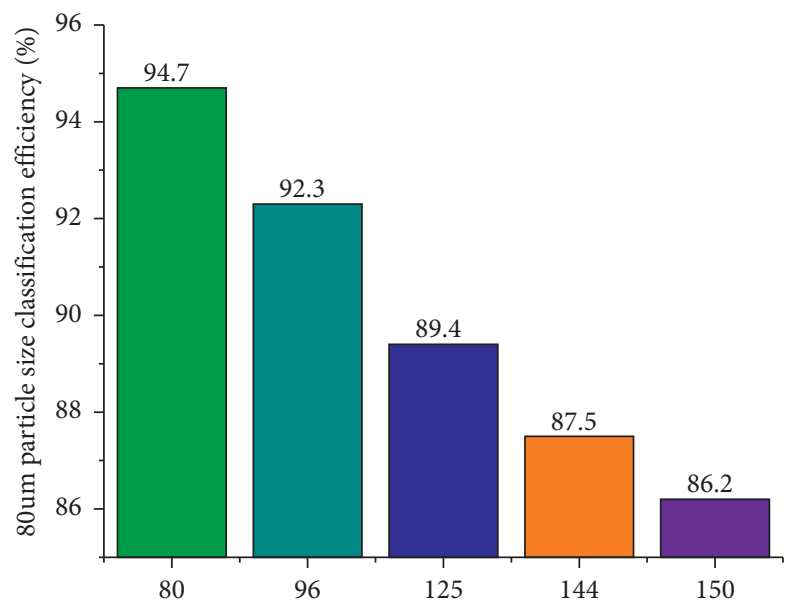

(d)

FIGURE 16: The relations between differential pressure, classification efficiency, and five groups of blades including (a) the pressure difference of the whole machine, (b) the pressure difference of the separator, (c) the $45 \mu \mathrm{m}$ particle size classification efficiency, and (d) the $80 \mu \mathrm{m}$ particle size classification efficiency. The results of the same group of blade analysis are shown in the same color.

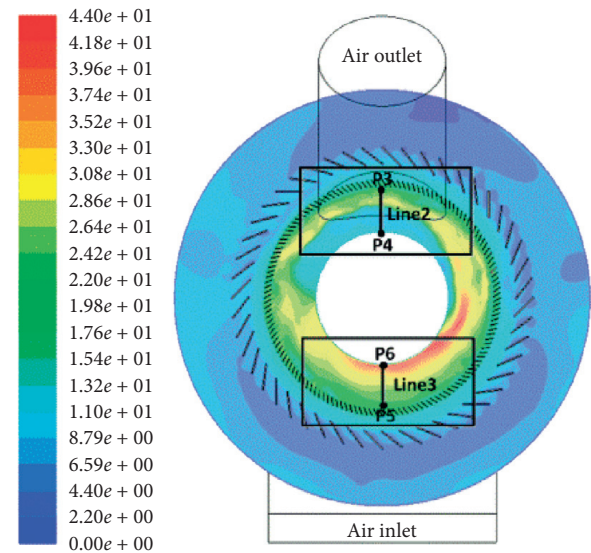

Figure 17: Velocity nephogram $(\mathrm{m} / \mathrm{s})$ of $Y=3.4 \mathrm{~m}$ section. 

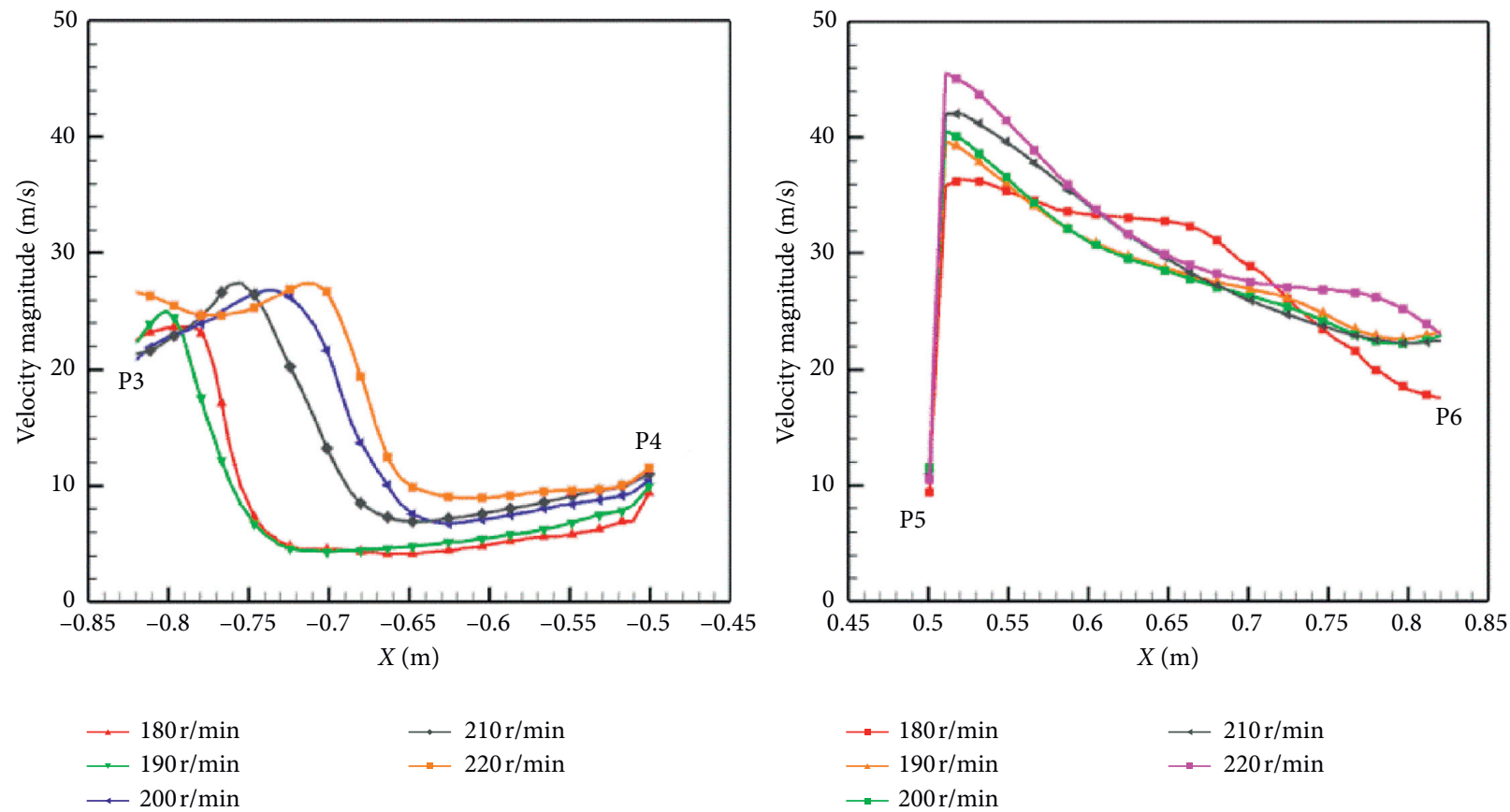

Figure 18: Speed curve of (a) Line 2 and (b) Line 3 at different speeds. The blade shape and number are TypeIand 144, respectively.

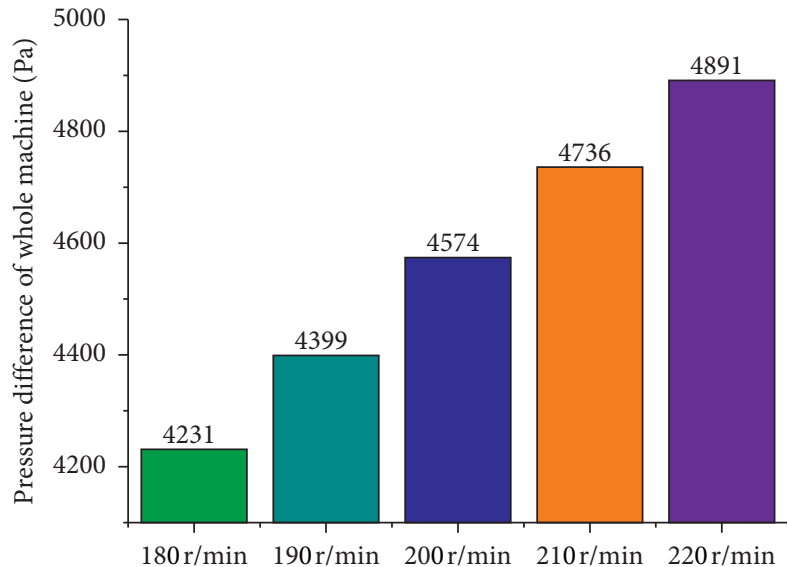

(a)

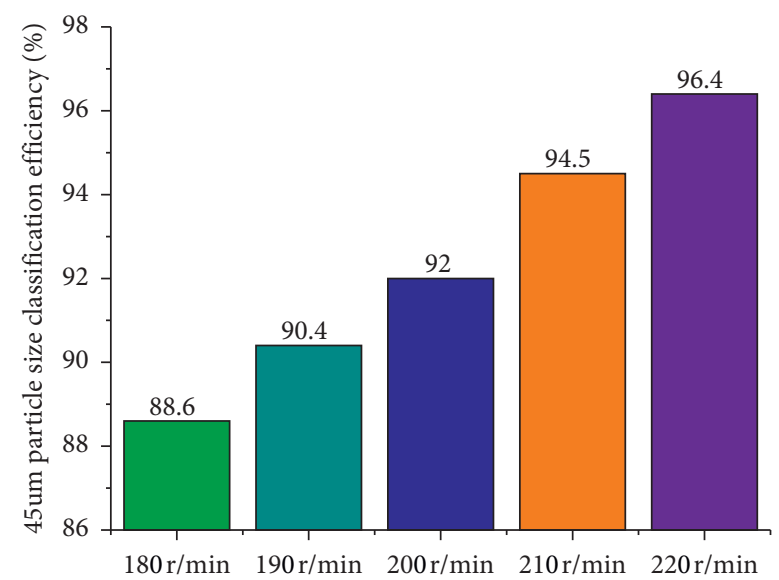

(c)

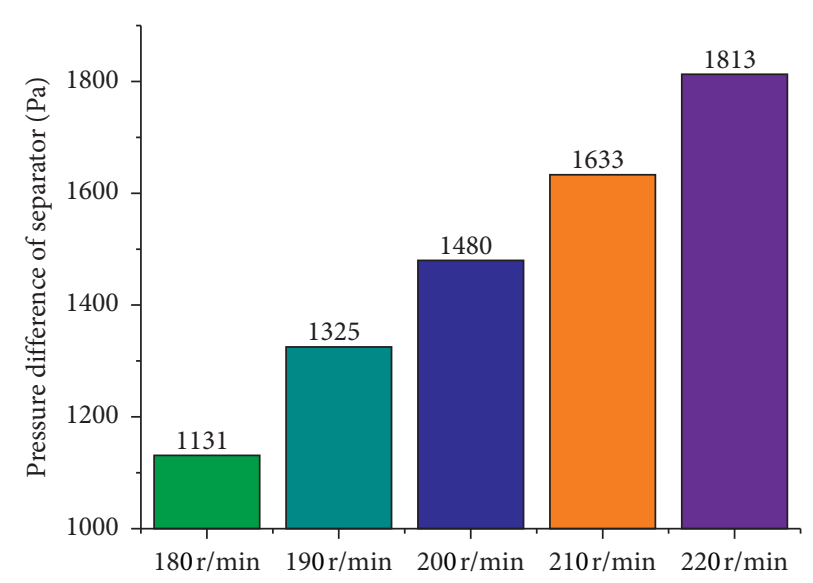

(b)

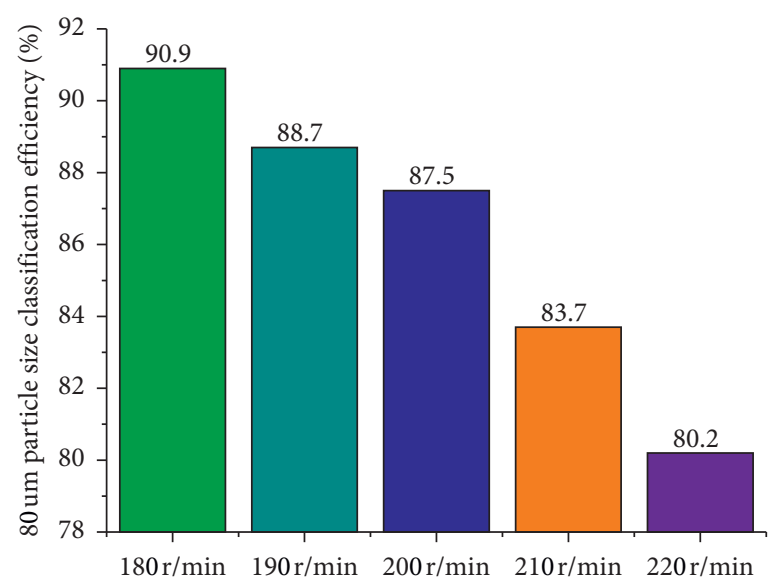

(d)

FIGURE 19: The relations between differential pressure, classification efficiency, and five groups of rotating speed. (a) The pressure difference of the whole machine. (b) The pressure difference of the separator. (c) The $45 \mu \mathrm{m}$ particle size classification efficiency. (d) The $80 \mu \mathrm{m}$ particle size classification efficiency. The results of the same group of blade analysis are shown in the same color. 


\section{Conclusions}

In this work, a numerical model focusing on an industryused vertical roller mill has been established based on the CFD method combining the continuous model with DPM. By comparing the particle size distribution and the differential pressure between the modeling results and the plant data, good agreements are obtained which means that the adopted numerical model is correct and at least reasonable. The flow characteristics in the mill have been systematically discussed from multiple aspects including velocity field, pressure field, and discrete phase distribution. The influences of the shape, number, and rotation speed of blades on the flow and classification performance of the vertical roller mill have been analyzed comprehensively. The simulation results show that some vortices exist in many parts of the mill and the blades are of great significance on the flow behaviors and classification performance.

In the simulated blade types, Type IV could not only have relatively high classification efficiency but also can effectively reduce the pressure difference of the separator and the whole equipment. The classification efficiency is proportional to the blade rotation speed as well as the blade number when the particle size is less than $45 \mu \mathrm{m}$. However, too high classification efficiency would need too excessive pressure difference, resulting in more power consumption. The parameters need to be defined properly under comprehensive consideration in practical applications. The results on the characteristics of flow field distribution and classification performance could give helpful suggestions to structure optimization and process optimization of a vertical roller mill, which is vital for ensuring product quality and reducing energy consumption.

\section{Data Availability}

The data used to support the findings of this study are included within the article.

\section{Conflicts of Interest}

The authors declare no conflicts of interest.

\section{Acknowledgments}

The authors would like to thank Wuhan University of Technology Cooperation Project (No. WHUT-20192h0067) for the financial support of this work.

\section{References}

[1] D. Altun, H. Benzer, N. Aydogan, and C. Gerold, "Operational parameters affecting the vertical roller mill performance," Minerals Engineering, vol. 103-104, pp. 67-71, 2017.

[2] J. Harder, "Grinding trends in the cement industry," ZKG International, vol. 63, no. 4, pp. 67-71, 2010.

[3] J. Jesweit and A. Szekeres, "Energy consumption in mining comminution," Procedia CIRP, vol. 48, pp. 140-145, 2016.

[4] R. A. C. Varas and M. A. Valenzuela, "Empirical determination of the effect of lifter wear in mill power for dry grinding," IEEE Transactions on Industry Applications, vol. 53, no. 3, pp. 2621-2627, 2017.

[5] K. Holmberg, P. Kivikytö-Reponen, P. Härkisaari, K. Valtonen, and A. Erdemir, "Global energy consumption due to friction and wear in the mining industry," Tribology International, vol. 115, pp. 116-139, 2017.

[6] H. Usman, P. Taylor, and D. E. Spiller, "The effects of lifter configurations and mill speeds on the mill power draw and performance," in Proceedings of the 1st International Process Metallurgy Conference, vol. 1805, pp. 10-16, Bandung, Indonesia, November 2017.

[7] Y. W. Sun, X. B. Zen, X. F. Yang, C. Y. Zhang, and Y. Q. Zhou, "Analysis of flow field in vertical roller mill chamber based on turbulent models," in Proceedings of the International Conference on Environmental Science and Information Application Technology (ESIAT 2009), Wuhan, China, July 2009.

[8] C. Bhasker, "Numerical simulation of turbulent flow in complex geometries used in power plants," Advances in Engineering Software, vol. 33, no. 2, pp. 71-83, 2002.

[9] H. B. Vuthaluru, V. K. Pareek, and R. Vuthaluru, "Multiphase flow simulation of a simplified coal pulveriser," Fuel Processing Technology, vol. 86, no. 11, pp. 1195-1205, 2005.

[10] H. J. Dou, X. T. Chai, and W. H. Nie, "Numerical study of the flow field in a vertical roller mill," Applied Mechanics and Materials, vol. 52-54, pp. 659-663, 2011.

[11] X. B. Ze, Y. W. Sun, W. Wang, J. L. Nie, and K. Mao, “Analysis of gas-solid coupling flow field in a vertical roller mill under different gas fluxes," Proceedings of the Institution of Mechanical Engineers, Part E: Journal of Process Mechanical Engineering, vol. 225, no. 1, pp. 20-28, 2011.

[12] Q. Huang, J. Liu, and Y. Yu, "Turbo air classifier guide vane improvement and inner flow field numerical simulation," Powder Technology, vol. 226, pp. 10-15, 2012.

[13] W. Ren, J. Liu, and Y. Yu, "Design of a rotor cage with nonradial arc blades for turbo air classifiers," Powder Technology, vol. 292, pp. 46-53, 2016.

[14] R. Liu, J. Liu, and Y. Yu, "Effects of axial inclined guide vanes on a turbo air classifier," Powder Technology, vol. 280, pp. 1-9, 2015.

[15] K. V. Shah, R. Vuthaluru, and H. B. Vuthaluru, "CFD based investigations into optimization of coal pulveriser performance: effect of classifier vane settings," Fuel Processing Technology, vol. 90, no. 9, pp. 1135-1141, 2009.

[16] C. Eswaraiah, R. K. Soni, S. K. Tripathy, and L. O. Filippov, "Particle classification optimization of a circulating air classifier," Mineral Processing and Extractive Metallurgy Review, vol. 40, no. 5, pp. 314-322, 2019.

[17] H. Li, Y. He, F. Shi et al., "Performance of the static air classifier in a vertical spindle mill," Fuel, vol. 177, pp. 8-14, 2016.

[18] Y. Zeng, S. Zhang, and M. Li, "Numerical simulation of a flow field in a turbo air classifier and optimization of the process parameters," Processes, vol. 8, no. 2, p. 237, 2020.

[19] M. E. Caliskan, I. Karagoz, A. Avci, and A. Surmen, "Investigation into the effects of various parameters on the performance and classification potential of a cyclone classifier," Powder Technology, vol. 356, pp. 102-111, 2019.

[20] H. K. Versteeg and W. Malalasekera, An Introduction to Computational Fluid Dynamics: The Finite Volume Method, Pearson Education, New York, NY, USA, 1st edition, 1995.

[21] T. J. Napier-Munn, Mineral Comminution Circuits, Julius Kruttschnitt Mineral Research Centre, Queensland, Australia, 1996. 
[22] C. L. Wu, K. Nandakumar, A. S. Berrouk, and H. KruggelEmden, "Enforcing mass conservation in DPM-CFD models of dense particulate flows," Chemical Engineering Journal, vol. 174, no. 1, pp. 475-481, 2011.

[23] S. A. Morsi and A. J. Alexander, "An investigation of particle trajectories in two-phase flow systems," Journal of Fluid Mechanics, vol. 55, no. 2, pp. 193-208, 1972. 